\title{
Hacking education. A formação entre a abertura e a tecnologia
}

\author{
Mario Pireddu
}

\section{Resumo}

Todas as instituições de ensino, incluindo escolas e universidades, baseiam-se no uso das tecnologias. Estas não são apenas ferramentas, mas, mais propriamente, meios de comunicação que têm trabalhado por um longo tempo como verdadeiros tradutores de experiências. Tem sido assim tanto para a exposição oral quanto para o livro-texto, com consequências de sua relevância educacional sobre os métodos de construção e tradução do conhecimento. As tecnologias de comunicação digital, em especial, os ambientes de rede, que estão afetando a maioria das formas de relações sociais e de intercâmbio de conhecimento, oferecem-se, cada vez mais, como arquiteturas para a participação do usuário, colocando novos desafios para os sistemas de ensino. A construção da internet e da web mostra uma abordagem diferente para a aprendizagem e para a promoção da criatividade: a cooperação horizontal e a comunicação aberta são as bases da cultura hacker, que tem sido o terreno fértil para as mais importantes inovações tecnológicas das últimas décadas. Essas práticas e esses valores, também devidamente modificados e atualizados, podem funcionar como um guia para uma reformulação radical dos sistemas de ensino.

Palavras-chave: Educação. Tecnologia. Abertura. Hacker. Mídia.

O que se considera em ética não é qualquer ação tomada individualmente, mas uma disposição para agir de uma certa maneira.

Paul Ricoeur

I have never let my schooling interfere with my education Mark Twain

Academia is to knowledge what prostitution is to love; close enough on the surface but, to the nonsucker, not exactly the same thing Nassim Nicholas Taleb

Our educational system is totally rearview $m$ irror. Marshall McLuhan

Recebido: 20/04/2013 - Aprovado: 23/06/2013

http://dx.doi.org/10.5335/rep.2013.3553

Pesquisador do Departamento de Ciências da Educação da Universidade de Roma Tre. E-mail: mario.pireddu@uniroma3.it. 
Quando falamos sobre o mundo e a filosofia hacker, é fácil ver como - também devido ao mau hábito de jornalistas e acadêmicos - a imaginação representa algo obscuro, impenetrável e fechado. A realidade, como se sabe, é que a filosofia e a ética hacker têm mais a ver com a abertura do que com o fechamento. Entre os significados do verbo to hack, há uma referência à ferramenta que se utiliza para cortar, abrir algo em várias partes. A filosofia e a ética hacker têm a ver com o código aberto, por exemplo, mas, mais geralmente, com o conceito de openness. Hoje, ouvimos, frequentemente, os termos "dados abertos", “acesso aberto", "governo aberto", "educação aberta”.

John Willinsky, da Universidade de Stanford, na Open Education Conference, realizada em Vancouver em outubro passado, disse que a killing application para a educação realmente existe quando aplicamos nossas lições para as nossas comunidades, "para que a aprendizagem acadêmica contribua para o crescimento de bibliotecas públicas e do conhecimento público da comunidade". Dessa forma, de acordo com Willinsky, só em sendo assim a educação pode ser "aberta"1.

Para educação aberta, aqui, referimo-nos apenas aos Massive Open Online Courses (MOOCs) - traduzidos por Cursos Online Massivos e Abertos -, como Coursera, edX, Udacity, Canvas Network, Futurelearn etc., ou aos open textbooks - livros didáticos abertos. Dos primeiros, poderíamos falar por um longo tempo, e nós sabemos que estes crescem no número de cursos disponíveis e no de assinantes em todo o mundo. A equipe de edX - consórcio de Harvard, MIT e outras universidades - programou a disponibilidade da plataforma MOOC de edX com código aberto em junho de 2013, a qual, portanto, poderá ser baixada gratuitamente e utilizada por todos. Quanto aos segundos, open textbooks, sabemos que há estudos em todo o mundo para promover a sua propagação (Califórnia nos Estados Unidos, British Columbia no Canadá, há também algo semelhante na Itália etc.), bem como algumas experiências originais como o Textbook hack-a-thon. Um exemplo vem da Finlândia, provavelmente, não por coincidência, o país onde foi criado o Linux ${ }^{\circledR}$ : em setembro de 2012, um grupo de mais de 30 pessoas entre professores, alunos e pesquisadores produziu em apenas três dias (one-weekend schoolbook sprint) um livro de matemática para a escola superior finlandesa, destinado a cerca de 30 mil alunos.

A iniciativa quis demonstrar que criar um livro para a escola pode ser uma experiência de colaboração livre e eficiente, e não a escrita individual lenta de um autor pago ${ }^{2}$. De acordo com Tommi Sottinen, professor de Business Mathematics na Universidade de Vaasa, o mais importante no projeto é a abertura (openness) e a transparência (transparency) ${ }^{3}$. O líder do projeto, Vesa Linja-aho, reforça que o conteúdo aberto de alta qualidade reduziu os custos que os alunos devem desembolsar para estudar. 
Educação aberta significa não somente "recursos educacionais abertos" (REA ou OER), embora existam experiências muitas vezes interessantes e úteis nesse campo. Desses recursos, bem como os MOOCs, geralmente indicamos o custo livre (ou, pelo menos, baixo). Isso é certamente verdade, mas a economia é, de fato, o aspecto mais importante do assunto? As mudanças que a educação está experimentando no mundo são realmente apenas uma questão de economia? Ou essas mudanças afetam, também, transformações de âmbito antropológico, cultural, social?

Na TEDx University of Nevada, recentemente, um menino de 13 anos de idade , Logan LaPlante, realizou um discurso bem recebido. A apresentação de Logan foi intitulada Hackschooling, ou "a educação como uma experiência saudável e feliz". Logan, em 15 minutos , convida todos a repensarem a educação para que a saúde, a felicidade, a sabedoria e a criatividade tornem-se prioridade. Felicidade e criatividade são o foco de sua educação ${ }^{4}$.

Logan explica que seu herói é Shane McConkey, grande esquiador, freerider e base jumper canadense (McConkey, infelizmente, faleceu há alguns anos nas Dolomitas, na Itália, durante a execução de um de seus saltos), que ele chama de hacker: não é um hacker de computador, mas um ski-hacker. O que Logan diz é que os hackers são inovadores: são pessoas que desafiam os sistemas de todos os tipos e procuram mudá-los para torná-los diferentes, melhores. Afirma, ainda, que está crescendo em um mundo que precisa de mais pessoas parecidas com o que ele chama de "hacker's mind-set", e não apenas no âmbito da tecnologia: tudo pode ser hackeado, o skiing e, também, a educação. "I hack my education", Logan diz, "eu experimento o que aprendo: é como um remix ou um mashup flexível do aprendizado [...] o hackschooling pode ser usado por todos, incluindo escolas tradicionais. Eu entendo que, quando você está motivado para aprender algo, você pode fazer muito mais em menos tempo"s.

Voltemos, agora, ao conceito de educação aberta. Ivan Illich, em seu livro A desescolarização da sociedade, fala sobre o uso aberto e exploratório das competências adquiridas pelas pessoas e sobre a "educação liberal". Gardner Campbell, diretor de inovação e desenvolvimento profissional no Virginia Tech Institute, afirma que é mais interessante falar sobre o verbo "abrir" (opening), do que sobre o adjetivo "aberto" (open). Um verbo, e não um adjetivo: é importante, portanto, que se refere a algo dinâmico, não estático ${ }^{7}$.

"Abrir": a etimologia latina da palavra refere-se ao descerrar, à ação de remover os impedimentos e os obstáculos, mas também se refere ao fazer algo visível, evidente. Então, libertar e fazer algo claro. Uma educação aberta é, portanto, uma educação livre e visível? Vamos dar um passo atrás, novamente a partir da etimologia. A etimologia da palavra educação refere-se ao verbo educare, geralmente 
entendido como ajudar, com disciplina adequada, para desenvolver as boas inclinações da alma, que significa levar para fora, ou liberar o homem de defeitos originais da natureza bruta. Mais uma vez, referem-se a conceitos como "criar" e "instruir".

Como observado por Ivan Illich, o termo "educação" é relativamente recente: a educação das crianças é mencionada pela primeira vez em francês, em um documento datado de 1498. O ano em que Erasmus estabeleceu-se em Oxford, Savonarola foi queimado na fogueira e Dürer gravou o Apocalipse. Em inglês, o termo aparece pela primeira vez em 1530. Ainda em 1632, o espanhol Lope de Vega escreve sobre educação como se fosse uma novidade. No início do século XVII, começou a afirmar-se a ideia de um homem que nasce incapaz para a sociedade, e, portanto, precisa de uma educação.

Há outra leitura do verbo educĕre que parece mais adequada e diz respeito ao trazer para fora o que está dentro, melhorar e ampliar o que é positivo em todos nós. A edição em português do Wikipedia define que "educação engloba os processos de ensinar e aprender" (LIH, 2009). A edição italiana há muito definiu a educação como "o ato, o efeito de educar, maneira de comportar-se de forma correta e urbana nas relações sociais" (WIKIPEDIA). Isso foi em setembro de 2008, e, mesmo que este verbete, educação, já tenha sido modificado um pouco, permanecem referências urbanas. O que leva os editores da Wikipedia a usarem os conceitos "correto" e "urbano" para descrever os processos educacionais?

A cidade é o ambiente que permitiu ao homem viver em um mundo de objetos criados por ele mesmo, tornar-se educado e, a partir disso, dominar outros seres vivos e elevar a sua própria espécie. O modelo da cidade moderna foi fundado retomando modelos gregos e latinos da pólis e da civitas. Na Itália medieval e em seguida renascentista, um dos exemplos mais conhecidos é a cidade de Palmanova. No dicionário italiano de sinônimos, "cidadão" equivale a "elegante, refinado, educado". O cidadão é, portanto, o homem que lê, que informa e discute, o homem que foi educado e higienizado (lembre-se a Declaração dos Direitos do Homem e do Cidadão, de 1789).

A construção do habitat urbano também está intimamente relacionada com a cidadania e a adoção de novas tecnologias para o trabalho, a troca, a comunicação, a transmissão de conhecimento. A figura do cidadão ideal - pessoa totalmente desenvolvida e educada - vincula-se, em nossa cultura, ao conceito de cidade ideal. Naturalmente, nenhuma cidade ideal nunca se materializou, mesmo no caso de cidades criadas a partir do zero para esse fim. O sociólogo e urbanista estadunidense Lewis Mumford (1961) lembrou-nos que a paideia, a formação do homem grego, ganha vida em uma pólis, que existe potencialmente como uma ideia, mas nunca como realização em tijolo ou mármore. 
Sabemos que a vida real está em contraste com a abstração e a idealização, encontramos, já na República de Platão, um ideal educativo negativo, restritivo, autoritário, que é recorrente no discurso utópico sobre a cidade e a educação. Não há poetas, amantes da música, não há solicitude paterna ou materna, não há mistura de ofícios e profisssões, não há luxo, nenhuma relação com os estrangeiros.

As ocupações manuais são de natureza desprezível na República: as classes superiores de filósofos, homens de cultura exercem o poder político sobre o restante da população, que deve ser treinada e subjugada para a transformação de cada pessoa em súdito disciplinado. É importante notar, aqui, que normalmente usamos a palavra "disciplina" para nos referirmos tanto à obediência como às disciplinas escolares.

Em resumo, o sistema fechado da cidade ideal é um sistema de controle da vida, criado em abstrato por um pensamento formal que regula tudo e que o torna geometricamente harmonioso, uma espécie de destino que atende a uma realização paradigmática em Celebration City, gated community, criada por Disney. É sobre essa idealização que se desenvolveu, ao longo do tempo, uma concepção particular de educação: por um lado, a recuperação da ideia da pólis grega, cidade harmônica e ordenada, no modelo de municípios italianos; por outro, a rejeição da civitas romana, a cidade que está crescendo continuamente e além de suas fronteiras, celebrada há mais de um século na pintura La città che sale, do artista futurista Boccioni. Uma cidade que se tornou, com o tempo, a metrópole e a megalópole.

A civitas romana não é uma forma em equilíbrio imóvel. Para os romanos, a cidade é móvel, não está parada; inclusive, um dos epítetos mais frequentes na fase final da civilização romana é Roma mobilis (CACCIARI, 2004). O que une pessoas tão diversas como as de Roma não é uma suposta origem comum ou ancestralidade, como na pólis grega, mas um objetivo comum, o futuro da cidade e de seus habitantes. A civitas não era, na concepção romana, um fundamento originário que devia ser restaurado, mas uma meta que devia ser alcançada.

A idealização de sujeito civilizado/incivilizado (alto/baixo, superior/inferior, entre outros dualismos) está desde sempre dentro do microcosmo da cidade, onde assume a forma de sujeito alfabetizado versus analfabeto, educado versus sem instrução etc. Elites e burocratas estatais do século XIX parecem temer o caos da metrópole, sua desordem e a mistura imprevisível de pessoas (ricos e alfabetizados, pobres e não alfabetizados). Em vista disso, criam para os jovens, através dos sistemas educativos, locais separados do mundo, lugares para reproduzir, em série, cidadãos cada vez mais alfabetizados, mais formados (quem sabe formatados!), quase exclusivamente com base em um único meio considerado nobre e silencioso: o livro. 
Em síntese, cidade, idealização, livro, isolamento, a educação, tudo como uma abstração. Ivan Illich expressou esse conceito claramente ao dizer que "os jovens são alienados a partir das escolas, que os isolam" (1971). E em 1969, McLuhan argumentou que as crianças dos anos 60 do século XX estavam atordoadas com as instituições educacionais, que eram as mesmas do século XIX, onde a informação era escassa, mas ordenada e estruturada em fragmentos, padrões classificados, disciplinas, tudo de acordo com os ritmos e princípios fordistas.

A lógica do espelho retrovisor, de McLuhan, aplicada à educação, conduz a que, diante de cada inovação tecnológica e social, sejamos levados a observar o atual, de acordo com a lógica anterior à própria mudança, ou a "retroceder no futuro" (MCLUHAN; FIORE, 1967, p. 94). O estudioso canadense cunhou o conceito de "espelho retrovisor", refletindo sobre as mudanças culturais e sensoriais de seus alunos de pós-graduação (LAMBERTI, 2000), para explicar as mudanças relacionadas com a introdução de novas tecnologias e novas mídias de comunicação e transmissão do conhecimento. Confrontado com situações novas, como em condições de transição, muitas vezes tentamos impor a forma do velho para o novo conteúdo, de "forçar os novos meios de comunicação para obrigá-los a fazer o trabalho dos velhos" (MCLUHAN; FIORE, 1967, p. 94).

Essa é, claramente, uma defesa ou uma forma de desconfiança. Envolvidos na trama de um ambiente de mídia feito por informação infinita e instantânea, muitos educadores têm dificuldade de compreender um fato: para o aluno, é cada vez menos importante saber, armazenar ou recuperar informações, e é cada vez mais importante ser capaz de encontrar, classificar, analisar, compartilhar, discutir, criticar e criar informações. Só dessa forma é possível falar de educação aberta.

Ainda usando a metáfora da cidade, o aspecto mais interessante da cidade-canteiro, proposta pelo arquiteto futurista Antonio Sant'Elia, reside em uma descrição sem precedentes do ambiente urbano metropolitano como "natureza construída", e não como uma ruína de um passado glorioso. Uma cidade que não é pensada como eterna, que não é construída para durar: a cidade dos futuristas não realiza a harmonia de proporções clássicas, mas a mudança constante e o consumo real do ambiente urbano pelas gerações que vivem lá no tempo.

A arquitetura contemporânea discute esses mesmos temas, e que não são apenas assuntos de planejamento urbano. A atenção para a natureza construída e a artificialização da realidade, o papel da tecnologia no habitat humano, o futuro como algo a ser alcançado e não a temer, a mudança como sistema são temas que se relacionam intimamente com nossa forma de compreender os processos educacionais e a maneira como organizamos nossos meios de comunicação.

$\mathrm{Na}$ Europa, é ainda forte a oposição de muitos intelectuais, educadores e estudiosos da comunicação para com as formas de expressão associadas com os meios 
de comunicação não tipográficos, como audiovisuais no século XX e, em seguida, tecnologias e redes digitais. Uma postura fechada e pré-concebida que tem todos os traços do conservadorismo e do idealismo e que se traduz em uma "forma de resistência através das tecnologias do alfabeto" (FRASCA, 2005). É o que Roberth Harris chama de "preconceito tipográfico" (1986), profundamente enraizado na educação europeia, que sempre venerou mais a palavra escrita do que a falada: maior respeito para o livro do que para os outros meios de comunicação, como cinema, televisão, jogos de vídeo, mídia digital e interativa, mídias sociais etc.

As implicações sobre o nível educacional de uma abordagem conservadora para a interpretação das mídias não tipográficas quase sempre resultam na construção de sistemas educacionais criados em torno de uma única tecnologia salvadora. A aprendizagem institucionalizada torna-se uma espécie de refúgio ou tábua de salvação ideal no implacável e dissipador naufragar contemporâneo.

Uma cultura de educação baseada no ensino e na produção fordista, e não na própria aprendizagem, é, de muitas maneiras, a imagem espelhada do que se conhece na teoria da comunicação como bullet theory (teoria da bala ou teoria da agulha hipodérmica). De acordo com essa abordagem teórica, as pessoas são agregados homogêneos, indistinguíveis, mesmo quando os componentes individuais vêm de origens e diferentes grupos sociais. Nessa visão, os indivíduos são anônimos e isolados, e a educação é meramente a passagem de informação de uma fonte a uma massa de indivíduos.

Esse parêntese sobre a educação e a cidade serviu para enfatizar, por um lado, a estreita relação entre a escrita e a cidade e, por outro, o excesso de idealização que faz coincidir, muitas vezes, a educação formal e o domínio da escrita. Então, para retornar às nossas perguntas iniciais, que tipo de educação temos na era da cidade expandida, da cidade das redes e dos fluxos (termo caro a Manuel Castells)?

Se pensamos sobre a chamada horizontalidade das redes, notamos que um achatamento crescente de hierarquias também é perceptível na esquizofrênica arquitetura cotidiana da cidade contemporânea: são sobrepostos, misturados e mencionados todos os tipos de estilos, de épocas diversas, há uma sensação de fragmentação e (com) presença de espaço e tempo. Isso traz à mente a metáfora da A catedral e o bazar, de Eric Raymond (1999), que usa dois lugares urbanos tão diferentes para explicar a lógica cultural do Linux ${ }^{\circledR}$. Novos espaços, novas formas, novas mídias, novas comunicações, novos conhecimentos. Nas cidades, hoje, vivem juntos uma pluralidade de códigos e uma variedade de formas de expressão, uma desordem (WEINBERGER, 2007), um terreno fértil para novas criações e experimentações inéditas.

Como resultado desse processo, estamos testemunhando o acalorado debate que caracteriza a reflexão pedagógica atual e vendo, de um lado, as posições daque- 
les que ainda aspiram a ter, na cidade rumorosa, uma escola silenciosa, uma alternativa ao mundo, e, de outro, aqueles que veem como um elemento indispensável para a educação contemporânea o diálogo entre a escola e o mundo.

Pensemos sobre o uso tipográfico e limitante da tecnologia da informação e das redes. Seymour Papert (1993) observou que a introdução de computadores nas escolas tem sido, desde o início, uma operação para neutralizar a força desestabilizadora das tecnologias digitais. Relegar o computador em uma sala dedicada à tecnologia da informação significa, mais uma vez, transformar as práticas de descoberta e interatividade em uma disciplina qualquer, regulada por programas ministeriais.

Contudo, os meios de comunicação são "matrizes e tecido da experiência" (SILVERSTONE, 1999), e as plataformas expressivas de novas mídias são capazes de dar às pessoas mais controle, mais espaço e mais iniciativa. Pense nas tecnologias que permitem editar, remixar e compartilhar áudio-vídeo, wikis, redes sociais, jogos de vídeo: mídias ligadas a percursos de aprendizagem comuns, as quais seria insensato deixar de fora das experiências educativas institucionais.

Henry Jenkins trabalhou extensivamente sobre o potencial de culturas da participação contemporâneas e sobre o Faça você mesmo (Do it your self). O mais interessante em suas pesquisas é a reconstrução de uma mudança de paradigma no referencial teórico e metodológico dos estudos de mídia (JENKINS, 2007, 2009). Nas culturas participativas, as pessoas são nós dentro de uma rede, capazes de interligar-se, de forma produtiva, com um número variável de outros nós. A divisão rígida do trabalho transforma-se em uma organização mais fluida do tempo e das funções, até o desaparecimento do planejamento rígido e pré-ordenado, típico do sistema taylorista.

O mesmo autor concentra-se na relação entre a lógica de produção e os mecanismos de participação que vêm de baixo, mostrando como o trabalho ativo de fãs, mediante estratégias complexas de apropriação e remixagem, tornou-se parte do processo de produção. O valor da participação, neste quadro teórico, não está unicamente nos produtos da indústria cultural ou do mercado, mas na vida diária e na educação.

O uso generalizado, de massa, mas também pessoal, da tecnologia de informação e comunicação ajuda a reconfigurar muitos aspectos de nossas vidas, incluindo as maneiras pelas quais criamos, consumimos, interagimos uns com os outros e aprendemos coisas novas. Os usuários adquirem cada vez mais capacidade autônoma de produção. Os usuários podem, agora livremente, manipular imagens, vídeos, música, sons, pedaços de software, videogames, dando origem a novos produtos, muitas vezes distantes das narrativas e intenções originais (em termos informáticos, podemos falar de "fork" culturais). 
Agora, nós sabemos que as críticas aos sistemas educacionais são tão antigas quanto a própria educação. Muitos autores têm apontado que a escola e a academia estão entre os poucos sistemas ainda estruturados a partir de métodos de fragmentação e divisão do trabalho, típicos das sociedades industriais, mas eles deveriam ser os lugares mais sensíveis e atentos à mudança e à inovação social e cultural ${ }^{8}$.

É útil, nesse ponto, uma comparação entre alguns breves textos que são muito diferentes, mas que possuem algo em comum. O primeiro consiste em um texto de 1919, de Giovanni Papini, cujo título provocativo é Vamos fechar as escolas: "[A escola,] para a sua necessidade formal e tradicionalista, tem frequentemente contribuído para petrificar o conhecimento e atrasar com obstrucionismo teimoso as mais urgentes revoluções intelectuais e reformas". A escola "não inventa conhecimento, mas vangloria-se de transmiti-lo [...]", transmite mal ou "transmitindo-o impede, na maior parte do tempo, torcendo e dissecando os cérebros receptores, a formação de outro conhecimento novo e melhor". A escola "ensina mal porque ensina as mesmas coisas da mesma maneira e com a mesma quantidade, sem ter em conta a diversidade infinita de raça, talento, idade, necessidades, etc.". Nas conclusões, afirma que "a escola é tão essencialmente antigenial que faz estúpidos não só os alunos, mas também os professores. Repita e repita todos os anos as mesmas coisas, eles se tornam muito mais estúpidos e inalteráveis do que eram no início" (1919, p. 5, 9 e 11).

O convite de Papini para fechar escolas, todas as escolas, da primeira para a última indica, no início do século XX, muitos dos pontos de distância da escola da realidade social. A partir da organização espaço-temporal e do isolamento físico das salas de aula, Papini fala de "imobilidade física mais antinatural", de "imobilidade do espírito obrigado a repetir em vez de pesquisar" e de "afogamento sistemático de cada personalidade, originalidade e iniciativa no mar negro dos programas uniformes" (1919, p. 7).

O segundo texto é composto de declarações de Marshall McLuhan sobre educação. "As escolas norte-americanas são prisões intelectuais [...]. Poucos alunos são capazes de sobreviver às torturas intelectuais de nosso sistema educacional. [...]. A nossa é uma época de sobrecarga de informações, e a única maneira de ter certeza de que as escolas não são prisões sem barras é começar do zero com novas técnicas e valores". "[...] O nosso sistema educativo é reacionário, orientado até valores e tecnologias do passado. Esse sistema está desatualizado e moribundo. O mero ensino e simples repetição dos fatos são irrelevantes. Se não adaptamos nosso sistema educacional aos valores e às necessidades dos alunos, vamos ver só mais evasão e caos. [...]. Para mergulhar no nosso ambiente, a arma privilegiada é a experiência direta. [...]. Com a cidade magnética todo o planeta se torna uma máquina educativa" (MCLUHAN, 1969, passim). 
O terceiro texto é uma definição de ensino contemporâneo, por Michael Wesch, antropólogo da Kansas University. Em seu trabalho, o autor lembra-nos de que a tecnologia é apenas um aspecto do problema que nós consideramos. Sim, está configurada como um fator facilitador, mas a atenção do educador deve ser dada à prática generalizada de compartilhamento e colaboração relacionada com o envolvimento dos usuários no ecossistema de mídias digitais interligados e nesse sentido devem ser entendidos os termos "Web 2.0" e "conteúdo gerado pelo usuário". Escreve Wesch, definindo o ensino institucional: "a prática continua, dia após dia, hora após hora, para se sentar e ouvir as autoridades para receber informações que serão regurgitadas mais tarde nos exames" (2009).

O norte-americano Curtis J. Bonk, autor do livro The world is open: how web technology is revolutionizing education, define a educação como "highly reactive and slow-moving industry" (2009, p. 18). Bonk fala sobre a rede e seu potencial disruptivo, usando o conceito de Web of learning, que inclui pessoas de todas as idades e estilos de todos os tipos, argumentando que estamos testemunhando hoje uma enorme liberação (uncover) do potencial humano. Abertura, transparência, liberação de potencial humano.

No mês de março 2013, foi publicado um texto cujo título tem a ver com o presente discurso. É Hacking your education, por Dale J. Stephens, que, em uma linha provocativa semelhante à de Papini, fala abertamente de unschooling e hackademic. Naturalmente, o conceito de unschooling refere-se à desescolarização de Illich, mas não tem a mesma força argumentativa. Stephens argumenta que o custo de cursos universitários aumenta enquanto diminui o seu valor e promove o abandono das instituições de ensino.

Ele deixou a escola e afirma ter escrito o livro para ajudar as pessoas a educarem-se. "Eu não sou contra as escolas", escreve ele, "eu sou a favor da escolha", e acrescenta que as escolas e universidades começaram a destruir-se sozinhas, para deixar lugar a empresas, grupos e organizações. "Hacking your education is a lifelong commitment", escreve Stephens. Ele argumenta que as três principais funções da universidade, a divulgação do conhecimento, a construção de comunidade, entrar em contato com as empresas, estão agora completamente substituídas. E fala, como Logan, de "sociedade feliz e saudável".

Ressalta que as respostas de algumas instituições de ensino, como a criação de MOOCs, por exemplo, já estão mostrando essa substituição e lembra as start-ups que surgiram nos últimos anos, muitas vezes nas incubadoras universitárias (Coursera, Udacity etc.). Ele mesmo fundou o UnCollege para interceptar as pessoas que não querem ir para a universidade: "nós não vamos dizer a ninguém o que tem que aprender. Em vez disso, vamos facilitar um ambiente de aprendizagem no qual [possa] explorar a si mesmo e ao mundo" (STEPHENS, 2013). 
Stephens incorpora muitos conceitos de Illich, que, na década de 1970, falava de livre participação em um ambiente significante e da necessária transferência de responsabilidade da instituição para o indivíduo. Wesch, por sua vez, fala a esse respeito de anti-teaching, algo como a transição da era de ensino para a era da aprendizagem. Illich argumentou a necessidade de libertar o acesso gratuito às coisas, libertar a transmissão das capacidades, libertar os recursos críticos e criativos das pessoas, libertar o indivíduo da obrigação de adaptar suas expectativas para os serviços oferecidos por uma profissão pré-estabelecida.

Também usa outra definição de Illich, a de tecelagens de aprendizagem (learning webs), que já em 1971 desenhou conceitos hoje muito atuais, como o peer-matching network. Em seu texto, Illich imaginava, trinta anos antes da web 2.0 e de muitas teorias do construtivismo e conectivismo, um sistema informatizado capaz de conectar as pessoas com base em seus interesses e capaz de fazer cada vez menos necessária a formação institucional.

Texturas de possibilidades, tecelagens de aprendizagem, novas redes para garantir a igualdade de possibilidades para aprender e ensinar, essas são as palavras de Illich, para quem é fora da escola que todos aprendem a viver, porque a escola opera uma divisão radical na realidade social, a educação é separada do mundo, o qual se torna não educacional. Agora, como educadores, se reconhecemos os limites da burocracia e trabalhamos para corrigir essas distorções, ainda devemos nos perguntar o seguinte: é possível reformar as instituições de ensino para que tenham um papel positivo na sociedade, e não desapareçam, como Stephens espera?

Porém, no final, temos de perguntar, já que estamos falando de redes, culturas e práticas participativas de produção colaborativa: como as referências à cultura hacker podem realmente nos ajudar?

Dentre as atividades da P2P Foundation está a de hacker scouts, para os quais hacking é simplesmente o ato de tomar algo, como um objeto ou uma ideia, e transformá-la de acordo com suas necessidades. Steven Levy, em seu livro Hackers, de 1984, escreveu que, para os hackers, as lições essenciais sobre sistemas e sobre o mundo podem ser aprendidas desmontando coisas, vendo como funcionam e usando esse conhecimento para criar novas coisas, ainda mais interessantes. Levy, também, insiste na liberdade, afirmando que, para os hackers, "toda a informação deve ser livre", e deve-se desconfiar de autoridade em favor da descentralização. Isso pode ser conseguido unicamente por meio de sistemas abertos (open systems) e absolutamente não burocratizados (LEVY, 1984).

A desconfiança de hackers em direção à burocratização dos sistemas está ligada a uma rejeição de regras arbitrárias, muitas vezes utilizadas para consolidar o poder e neutralizar os impulsos criativos. A ética hacker lembra-nos de algumas 
considerações de Illich: um hacker não deve ser julgado com base em qualificações, idade, raça ou posição, mas segundo a sua obra (judged by their hacking).

Outra sugestão é que a ética hacker associa-se à criatividade: "você pode criar arte e beleza em um computador" (hoje essa declaração soa como certa, mas o mesmo não se pensava em 1984), porque um computador pode mudar a vida para melhor. Criatividade, arte, beleza. Geralmente, aqueles que não conhecem a cultura hacker pensam unicamente em números, protocolos, cabos, redes de computadores.

Foi Pekka Himanen, no seu livro The hacker ethic and the spirit of the information age (2001), quem nos informou que o que move os hackers é a paixão. Também foi Himanen quem disse que a ética hacker espalha-se de computer hackers para grupos mais amplos de trabalhadores. E aqui nós estamos. A palavra grega skhole, além disso, indicava lazer, divertimento, tempo livre, uma vida em que as pessoas organizaram seu tempo combinando negócios com prazer. Isso é o que Himanem chama de a "domingação da sexta-feira" (the sundayization of friday).

Para concluir, entramos em uma era denominada de "pós-PC", e os dados reais sobre as vendas de PC mostram um declínio importante em 2012, em favor de dispositivos móveis, como smartphones e tablets. Agora, mais do que nunca, precisamos da cultura hacker, porque entramos em um mundo de walled gardens (jardins murados), um mundo em que a "abertura" é um termo amplamente usado na retórica corporativa, mas, ao mesmo tempo, é um exercício pouco praticado. Pensemos no Google, Apple, Amazon, Facebook etc.

Os educadores mais inovadores no presente e no futuro serão pessoas capazes de invadir o sistema atual e criar algo novo. Buckminster Fuller, arquiteto, inventor e escritor estadunidense, sentenciou: "você nunca irá mudar as coisas pela luta contra a realidade existente. Para mudar alguma coisa, é necessário construir um novo modelo que faz o modelo existente obsoleto".

Retomando as palavras de Michael Wesch, a beleza do momento em que vivemos é de que os novos meios de comunicação e da rede arrastaram nós educadores para um ambiente dinâmico, composto de perguntas e respostas, observações de tendências, compartilhamento de exposições e argumentos. Não há respostas fáceis, mas podemos pelo menos ser gratos pelas questões que esse ambiente estimula. 


\title{
Hacking education. Education between openness and technology
}

\section{Abstract}

\begin{abstract}
All educational institutions, including schools and universities, are based on the use of technologies. These are not just tools, but, more specifically, media that have been working for a long time as true translators of experience. It has been so for both, oral exposure and for the textbooks, with consequences of their educational relevance on the ways we build and translate knowledge. The ICTs and the network environments that are affecting most of the forms of social relations and knowledge interchange are, more and more, architectures for the participation of users, posing new challenges to education systems. The construction of the Internet and the web shows a different approach to learning and to the promotion of creativity: horizontal cooperation and open communication are the foundation of the hacker culture that has been the breeding ground for the most important technological innovations of recent decades. These practices and values, also properly modified and updated, can act as a guide for a radical overhaul of education systems.
\end{abstract}

Keywords: Education. Technology. Openness. Hacker. Media.

\section{Notas}

1 John Willinsky's keynote, entitled "Open for What? Open to What? Beyond Content," delivered on Day 2 of Open Ed 2012, Wednesday October 17, 2012, Vancouver, BC. Disponível em: $<$ http://www.youtube.com/watch?v=dYDpzIsI7aQ > . Accesso em: 27-09-2013.

2 Disponível em: <http://creativecommons.org/weblog/entry/34643>. Accesso em: 27-09-2013.

3 Disponível em: <http://www.youtube.com/watch?v=ThbUiky4AKA >. Accesso em: 27-09-2013.

4 "Think differently about education to make health, happiness, creativity, and wisdom a priority". Hackschooling makes me happy: Logan La Plante at TEDx University of Nevada. Disponível em: <http://www. youtube.com/watch?v=h11u3vtcpaY>. Accesso em: 27-09-2013.

5 "Ecologies of yearning", Gardner Campbell's keynote - Open Ed '12, October 16, 2012, Vancouver BC. Disponível em: <http://youtu.be/kIzA4ItynYw>. Accesso em: 27-09-2013.

6 Há um vídeo de Ken Robinson circulando na internet, intitulado "Do schools kill creativity?" <http://www. youtube.com/watch?v=iG9CE55wbtY>. Accesso em: 27-09-2013.

\section{Referências}

BENKLER, Y. The wealth of networks: how social production transforms markets and freedom. New Haven and London: Yale University Press, 2006.

"Sharing nicely": on shareable goods and the emergence of sharing as a modality of economic production. The Yale Law Journal, v. 114, p. 273-358, 2004.

BENNETT, S. et al. The "digital natives" debate: a critical review of the evidence. British Journal of Educational Technology, v. 39, n. 5, p. 775-786, 2008.

BONK, C. J. The world is open: how web technology is revolutionizing education. San Francisco: Jossey-Bass, 2009.

BUCKINGHAM, D. The media literacy of children and young people: a review of the literature. London: Centre for the Study of Children Youth and Media Institute of Education, University of London, 2005. 
CACCIARI, M. La città. Villa Verucchio (RN): Pazzini Editore, 2004.

COLLINS, A.; HALVERSON, R. Rethinking dducation in the age of technology: the digital revolution and schooling in America. New York: Teachers College Press, 2009.

FRASCA, G. La lettera che muore: la "letteratura" nel reticolo mediale. Roma: Meltemi, 2005.

GASSER, U.; PALFREY, J. Born digital: understanding the first generation of digital natives. New York: Basic Books, 2008.

HARRIS, R. The origin of writing. London: Duckworth, 1986.

HEW, K. F.; HARA, N. Knowledge sharing in online environments: a qualitative case study. Journal of the American Society for Information, Science and Technology, v. 58, Issue 14, p. 2310-2324, dec. 2007.

HIMANEN, P. The hacker ethic and the spirit of the information age. New York: Random House, 2001.

ILLICH, I. Deschooling society. London: Harper \& Row, 1971.

ITO, M. et al. Hanging out, messing around, geeking out: living and learning with new media. Cambridge: MIT Press, 2009.

JENKINS, H. Reconsidering digital immigrants. Confessions o Fan Aca-Fan, 5 dec. 2007. Disponível em: <http://www.henryjenkins.org/2007/12/reconsidering_digital_immigran.html>. Accesso em: 27-09-2013.

JENKINS, H. et. al. Confronting the challenges of participatory culture: media education for the 21st Century. Chicago: The MacArthur Foundation, 2009.

KVAVIK, R. B. Convenience, communications and control: how students use technology. In: OBLINGER, D.; OBLINGER, J. (Eds.). Educating the net generation. Washington DC: Educause, 2005. Disponível em: <http://net.educause.edu/ir/library/pdf/pub7101g.pdf>. Accesso em: 2709-2013.

LAMBERTI E. Marshall McLuhan. Milano: Bruno Mondadori, 2000.

LANKSHEAR, C.; KNOBEL, M. (Eds.). Digital literacies: new literacies and digital epistemologies. New York: Peter Lang Publishing, 2008.

LEVINSON, M. From fear to Facebook: one school's journey. Oregon: ISTE, 2010.

LEVY S. Hackers: heroes of the computer revolution. New York: Doubleday, 1984.

LIH, A. The Wikipedia revolution: how a bunch of nobodies create the world's largest encyclopedia. New York: Hyperion, 2009.

LIVINGSTONE, S. The changing nature and uses of media literacy. London: MEDIA@LSE Electronic Working Papers, 2003. Disponível em: <http://www.lse.ac.uk/media@lse/research/mediaWorkingPapers/pdf/EWP04.pdf>. Accesso em: 27-09-2013.

MCLUHAN, M. Playboy interview: Marshall McLuhan. Playboy, p. 26-63, mar. 1969.

MCLUHAN, M.; FIORE, Q. The medium is the massage: an inventory of effects. London: Penguin Books, 1967.

MOE, T. M.; CHUBB, J. E. Liberating learning: technology, politics, and the future of American education. San Francisco: Jossey-Bass, 2009.

MUMFORD, L. The city in history: its origins, its transformations, and its prospects. New York: Harcourt, Brace \& World, 1961.

OECD. New Millennium Learners. Initial findings on the effects of digital technologies on schoolage learners. OECD / CERI International Conference "Learning in the 21st Century: Research, Innovation and Policy”, Paris, 15-16 may 2008. 
O'REILLY, T. Web 2.0: compact definition. 2005a. Disponível em: <http://radar.oreilly.com/2005/10/web-20-compact-definition.html>. Accesso em: 27 set. 2013.

What is web 2.0: design patterns and business models for the next generation of software. 2005b. Disponível em: <http://oreilly.com/web2/archive/what-is-web-20.html>. Accesso em: 27 set. 2013.

PAPERT, S. The children's machine: rethinking school in the age of the computer. New York: Basic Books, 1993.

PAPINI, G. Chiudiamo le scuole. Viterbo: Millelire, 1992.

PRENSKY, M. H. Digital natives, digital immigrants. On the Horizon, MCB University Press, v. 9, n. 5, p. 1-6, oct. 2001.

. Sapiens digital: from digital immigrants and digital natives to digital wisdom. Innovate - Journal of Online Education, feb./mar. 2009. Disponível em: $<$ http://www.wisdompage.com/Prensky01.html>. Accesso em: 27-09-2013.

RAYMOND, E. The cathedral and the bazaar. Sebastopos (California): O'Reilly, 1999.

ROSEN, L. D. Rewired: understanding the igeneration and the way they learn. New York: Palgrave Macmillan, 2010.

SHAH, N.; ABRAHAM, S. Digital natives with a cause? A knowledge survey and framework. Hivos Knowledge Programme, Report 2009, San Francisco, p. 24, 2009. Disponível em: <http:// www.cis-india.org/research/dn/uploads/dnrep1>. Accesso em: 27-09-2013.

SHIRKY, C. Weblogs and the mass amateurization of publishing. Networks, Economics and Culture Mailing List, 03 oct. 2002. Disponível em: <http://www.shirky.com/writings/weblogs_publishing.html>. Accesso em: 27 set. 2013.

Power laws, weblogs, and inequality. Networks, Economics and Culture Mailing List, 08 feb. 2003. Disponível em: <http://www.shirky.com/writings/powerlaw_weblog.html>. Accesso em: 27 set. 2013.

SILVERSTONE, R. Why study the media? London: SAGE, 1999.

STEPHENS, D. Hacking your education: ditch the lectures, save tens of thousands, and learn more than your peers ever will. New York: Perigee, 2013.

TAPSCOTT, D. Grown up digital: how the net generation is changing your world. New York: McGraw Hill, 2009.

THINK differently about education to make health, happiness, creativity, and wisdom a priority. Hackschooling makes me happy: Logan LaPlante at TEDx University of Nevada. Disponível em: <http://www.youtube.com/watch?v=h11u3vtcpaY>. Accesso em: 27 set. 2013.

WEINBERGER, D. Everything is miscellaneous: the power of the new digital disorder. New York: Henry Holt, 2007.

WESCH, M. From knowledgable to knowledge-able: learning in new media environments. Academic Commons - New media technologies and the scholarship of teaching and learning, jan. 2009. Disponível em:

<http://www.academiccommons.org/2009/01/from-knowledgable-to-knowledge-able/>. Accesso em: 27 set. 2013. 